\title{
Comparison of Nonlinear Conjugate-Gradient Methods for Computing the Electronic Properties of Nanostructure Architectures ${ }^{\star}$
}

\author{
Stanimire Tomov ${ }^{1}$, Julien Langou ${ }^{1}$, Andrew Canning ${ }^{2}$, \\ Lin-Wang Wang ${ }^{2}$, and Jack Dongarra ${ }^{1}$ \\ 1 Innovative Computing Laboratory, \\ The University of Tennessee, \\ Knoxville, TN 37996-3450 \\ 2 Lawrence Berkeley National Laboratory, \\ Computational Research Division,
}

Berkeley, CA 94720

\begin{abstract}
In this article we report on our efforts to test and expand the current state-of-the-art in eigenvalue solvers applied to the field of nanotechnology. We singled out the nonlinear conjugate gradients (CG) methods as the backbone of our efforts for their previous success in predicting the electronic properties of large nanostructures and made a library of three different solvers (two recent and one new) that we integrated into the parallel PESCAN (Parallel Energy SCAN) code [3] to perform a comparison.
\end{abstract}

\section{Introduction}

First-principles electronic structure calculations are typically carried out by minimizing the quantum-mechanical total energy with respect to its electronic and atomic degrees of freedom. Subject to various assumptions and simplifications [5], the electronic part of this minimization problem is equivalent to solving the single particle Schrödinger-type equations (called Kohn-Sham equations)

$$
\begin{gathered}
\hat{H} \psi_{i}(r)=\epsilon_{i} \psi_{i}(r), \\
\hat{H}=-\frac{1}{2} \nabla^{2}+V
\end{gathered}
$$

where $\psi_{i}(r)$ are the single particle wave functions (of electronic state $i$ ) that minimize the total energy, and $V$ is the total potential of the system. The wave functions are most commonly expanded in plane-waves (Fourier components) up to some cut-off energy which discretizes equation (1). In this approach the

\footnotetext{
^ This work was supported by the US Department of Energy, Office of Science, Office of Advanced Scientific Computing (MICS) and Basic Energy Science under LAB03-17 initiative, contract Nos. DE-FG02-03ER25584 and DE-AC03-76SF00098.
} 
lowest eigen-pairs are calculated for $\hat{H}$ and the Kohn-Sham equations are solved self-consistently. For a review of this approach see reference [5] and the references therein. The computational cost of this approach scales as the cube of the number of atoms and the maximum system size that can be studied is of the order of hundreds of atoms. In the approach used in PESCAN developed by L-W. Wang and A. Zunger [9] a semi-empirical potential or a charge patching method [7] is used to construct $V$ and only the eigenstates of interest around a given energy are calculated, allowing the study of large nanosystems (up to a million atoms). The problem then becomes: find $\psi$ and $E$ close to a given $E_{r e f}$ such that

$$
H \psi=E \psi
$$

where $H$ represents the Hamiltonian matrix, which is Hermitian with dimension equal to the number of Fourier components used to expand $\psi$. The dimension of $H$ may be of the order of a million for large nanosystems. The eigenvalues $E$ (energy of state $\psi$ ) are real, and the eigenvectors $\psi$ are orthonormal.

In many cases, like semiconductor quantum dots, the spectrum of $H$ has energy gaps and of particular interest to physicists is to find a few, approximately 4 to 10 , of the interior eigenvalues on either side of the gap which determines many of the electronic properties of the system. Due to its large size $H$ is never explicitly computed. We calculate the kinetic energy part in Fourier space, where it is diagonal, and the potential energy part in real space so that the number of calculations used to construct the matrix-vector product scales as $n \log n$ rather than $n^{2}$ where $n$ is the dimension of $H$. Three dimensional FFTs are used to move between Fourier and real space. $H$ is therefore available as a procedure for computing $H x$ for a given vector $x$. Thus one more requirement is that the solver is matrix free. Finally, repeated eigenvalues (degeneracy) of approximately 3 maximum are possible for the problems discussed and we need to be able to resolve such cases to fully understand the electronic properties of our systems.

Currently, equation (2) is solved by a CG method as coded in the PESCAN package [9]. While this program works well for 1000 atom systems with a sizable band gap (e.g., $1 \mathrm{eV}$ ), it becomes increasingly difficult to solve for systems with (1) large number of atoms (e.g, more than 1 million); (2) small band gap, and where (3) many eigenstates need to be computed (e.g, more than 100), or to solve eigenstates when there is no band gap (e.g, for Auger or transport calculations). Thus, new algorithm to solve this problem is greatly needed.

The focus of this paper is on nonlinear CG methods with folded spectrum. The goal is to solve the interior eigenstates. Alternative for the folded spectrum transformation are shift-and-invert or fixed-polynomial [6]. Our choice of method is based on the highly successful current scheme [3] which has been proven to be efficient and practical for the physical problems we are solving. It will be the subject of other studies to further investigate the applicability of other alternatives like the Lanczos and Jacobi-Davidson method.

In Section 2 we describe the three eigensolvers investigated in the paper. We give our numerical results in Section 3, and finally, in Section 4, we give some concluding remarks. 


\section{Nonlinear CG Method for Eigenvalue Problems}

The conventional approach for problems of very large matrix size is to use iterative projection methods where at every step one extracts eigenvalue approximations from a given subspace $S$ of small dimension (see e.g. 2]). Nonlinear CG methods belong to this class of methods. Let us assume for now that we are looking for the smallest eigenvalue of the Hermitian operator $A$.

This eigenvalue problem can be expressed in terms of function minimization as: find the variational minimum of $F(x)=\langle x, A x\rangle$, under the constraint of $x^{T} x=I$, on which a nonlinear CG method is performed. The orthonormal constraint $x^{T} x=I$ makes the problem nonlinear.

In this section, we first give a description of the algorithms that we have implemented in our library, namely: the preconditioned conjugate gradient method (PCG), the PCG with $S=\operatorname{span}\{X, R\}$ method (PCG-XR), and the locally optimal PCG method (LOPBCG). Finally, we describe the spectral transformation that we use to get the interior eigen-values of interest.

\subsection{PCG Method}

In Table1, we give a pseudo-code of the PCG algorithm for eigen-problems. This is the algorithm originally implemented in the PESCAN code (see also [5, 8]).

Here $\mathrm{P}$ is a preconditioner for the operator $A, X$ is the block of blockSize column eigenvectors sought, and $\lambda$ is the corresponding block of eigenvalues. In

Table 1. PCG algorithm

\begin{tabular}{|c|c|}
\hline 1 & do $i=1$, niter \\
\hline 2 & do $m=1$, blockSize \\
\hline 3 & orthonormalize $\mathrm{X}(\mathrm{m})$ to $\mathrm{X}(1: \mathrm{m}-1)$ \\
\hline 4 & $\mathrm{ax}=\mathrm{AX}(\mathrm{m})$ \\
\hline 5 & do $\mathrm{j}=1$, nline \\
\hline 6 & $\lambda(\mathrm{m})=\mathrm{X}(\mathrm{m}) \cdot \mathrm{ax}$ \\
\hline 7 & if $\left(\|\mathrm{ax}-\lambda(\mathrm{m}) \mathrm{x}(\mathrm{m})\|_{2}<\right.$ tol .or. $\left.\mathrm{j}==\mathrm{nline}\right)$ exit \\
\hline 8 & $r_{j+1}=\left(I-X X^{T}\right)$ ax \\
\hline 9 & $\beta=\frac{\left(r_{j+1}-r_{j}\right) \cdot \operatorname{Pr}_{j+1}}{r_{j} \cdot \operatorname{Pr}_{j}}$ \\
\hline 10 & $d_{j+1}=-P r_{j+1}+\beta d_{j}$ \\
\hline 11 & $d_{j+1}=\left(I-X X^{T}\right) d_{j+1}$ \\
\hline 12 & $\gamma=\left\|d_{j+1}\right\|_{2}^{-1}$ \\
\hline 13 & $\theta=0.5\left|\operatorname{atan} \frac{2 \gamma \mathrm{d}_{j+1} \cdot \operatorname{ax}}{\lambda(\mathrm{m})-\gamma^{2} \mathrm{~d}_{j+1} \cdot \mathrm{A} \mathrm{d}_{j+1}}\right|$ \\
\hline 14 & $\mathrm{X}(\mathrm{m})=\cos (\theta) \mathrm{X}(\mathrm{m})+\sin (\theta) \gamma \mathrm{d}_{\mathrm{j}+1}$ \\
\hline 15 & $a x=\cos (\theta) a x+\sin (\theta) \gamma \mathrm{Ad}_{j+1}$ \\
\hline 16 & enddo \\
\hline 17 & enddo \\
\hline 18 & {$[\mathrm{X}, \lambda]=$ Rayleigh - Ritz on $\operatorname{span}\{\mathrm{X}\}$} \\
\hline 19 & enddo \\
\hline
\end{tabular}


Table 2. LOBPCG algorithm

\begin{tabular}{|ll|}
\hline 1 & do $i=1$, niter \\
2 & $\mathrm{R}=\mathrm{P}\left(\mathrm{A} \mathrm{X}_{\mathrm{i}}-\lambda \mathrm{X}_{\mathrm{i}}\right)$ \\
3 & check convergence criteria \\
4 & {$\left[\mathrm{X}_{\mathrm{i}}, \lambda\right]=$ Rayleigh - Ritz on $\operatorname{span}\left\{\mathrm{X}_{\mathrm{i}}, \mathrm{X}_{\mathrm{i}-1}, \mathrm{R}\right\}$} \\
5 & enddo \\
\hline
\end{tabular}

the above procedure, $X^{T} X=I$ is satisfied throughout the process. $\left(I-X X^{T}\right)$ is a projection operator, which when applied to $y$ deflates $\operatorname{span}\{X\}$ from $y$, thus making the resulting vector orthogonal to $\operatorname{span}\{X\}$. The matrix-vector multiplication happens at line 15. Thus there is one matrix-vector multiplication in each $j$ iteration. The above procedure converges each eigen-vector separately in a sequential way. It is also called state-by-state (or band-by-band in the physics community) method, in contrast to the Block method to be introduced next.

\subsection{LOBPCG Method}

Briefly, the LOBPCG method can be described with the pseudo-code in Table 2 Note that the difference with the PCG is that the $\mathrm{m}$ and $\mathrm{j}$ loops are replaced with just the blocked computation of the preconditioned residual, and the RayleighRitz on $\operatorname{span}\left\{\mathrm{X}_{\mathrm{i}}\right\}$ with Rayleigh-Ritz on $\operatorname{span}\left\{\mathrm{X}_{\mathrm{i}-1}, \mathrm{X}_{\mathrm{i}}, \mathrm{R}\right\}$ (in the physics community Rayleigh-Ritz is known as the process of diagonalizing $A$ within the spanned subspace, and taking the "blocksize" lowest eigen vectors). The direct implementation of this algorithm becomes unstable as $X_{i-1}$ and $X_{i}$ become closer and closer, and therefore special care and modifications have to be taken (see 4]).

\section{$2.3 \quad$ PCG-XR Method}

PCG-XR is a new algorithm that we derived from the PCG algorithm by replacing line 18 in Table 1 with

$$
18 \quad[\mathrm{X}, \lambda]=\text { Rayleigh }- \text { Ritz on } \operatorname{span}\{\mathrm{X}, \mathrm{R}\}
$$

The idea, as in the LOBPCG, is to use the vectors $R$ to perform a more efficient Rayleigh-Ritz step.

\section{$2.4 \quad$ Folded Spectrum}

Projection methods are good at finding well separated (non-clustered) extremal eigenvalues. In our case, we are seeking for interior eigenvalues and thus we have to use a spectral transformation, the goal being to map the sought eigenvalue of our operator to extremal eigenvalues of another one.

To do so we use the folded spectrum method. The interior eigenvalue problem $H x=\lambda x$ is transformed to find the smallest eigenvalues of $\left(H-E_{r e f} I\right)^{2} x=\mu x$. The eigenvalues of the original problem are given back by $\mu=(\lambda-s)^{2}$. 
The PCG algorithm in its folded form (FS-PCG) is described in [8]. To adapt the folded spectrum to LOBPCG (FS-LOBPCG), we have added three more block vectors that store the matrix-vector products of the blocks $X, R$, and $P$ with the matrix $H$. This enables us to control the magnitude of the residuals for an overhead of a few more axpy operations (otherwise we just have access to the magnitude of the residuals of the squared operator). Also the deflation strategy of LOBPCG is adapted in FS-LOBPCG, as the vectors are deflated when the residual relative to $H$ has converged (not $H^{2}$ ).

\section{$3 \quad$ Numerical Results}

\subsection{Software Package}

We implemented the LOBPCG 1, PCG, and PCG-XR methods in a software library. Currently, it has single/double precision for real/complex arithmetic and both parallel (MPI) and sequential versions. The library is written in Fortran 90. The folded spectrum spectral transformation is optional. The implementation is stand alone and meant to be easily integrated in various physics codes.

A test case is provided with the software. It represents a 5-point operator where the coefficients $a$ (diagonal) and $b$ (for the connections with the 4 closest neighbors on a regular 2D mesh) can be changed. In Table 1 the output of the test is presented. It is performed on a Linux Intel Pentium IV with Intel Fortran compiler and parameters $(a=8, b=-1-i)$. We are looking for the 10 smallest eigenstates, the matrix size is 20,000, and the iterations are stopped when all the eigencouples $(x, \lambda)$ satisfy $\|H x-x \lambda\| \leq$ tol $\|x\|$, with tol $=10^{-8}$.

Table 3. Comparison of the PCG, PCG-XR and LOBPCG methods in finding 10 eigenstates on a problem of size $20,000 \times 20,000$

\begin{tabular}{|c|r|r|r|}
\hline & PCG & LOBPCG & PCG-XR \\
\hline time $(\mathrm{s})$ & 37.1 & 61.7 & 20.2 \\
\hline matvecs & 3,555 & 1,679 & 1,760 \\
\hline dotprds & 68,245 & 137,400 & 37,248 \\
\hline axpys & 66,340 & 158,261 & 36,608 \\
\hline copys & 6,190 & 9,976 & 3,560 \\
\hline
\end{tabular}

In general LOBPCG always performs less iterations (i.e. less matrix-vector products) than PCG. This advantage comes to the cost of more vector operations (axpys and dot products) and more memory requirements. In this case, LOBPCG performs approximately 2 times more dot products for 2 times less matrix vector

\footnotetext{
${ }^{1}$ http://www-math.cudenver.edu/ aknyazev/software/CG/latest/lobpcg.m (revision 4.10 written in Matlab, with some slight modifications).
} 
Table 4. Comparison of FS-PCG, FS-PCG-XR and FS-LOBPCG methods in finding 10 eigenstates around the gap of quantum dots of increasing size

\begin{tabular}{l|crr}
\multicolumn{4}{c}{$(20 \mathrm{Cd}, 19 \mathrm{Se}) \mathrm{n}=11,331$} \\
& \# matvec outer & it & time \\
\hline FS-PCG(50) & 4898 & $(8)$ & $50.4 \mathrm{~s}$ \\
FS-PCG-XR(50) & 4740 & $(6)$ & $49.1 \mathrm{~s}$ \\
FS-LOBPCG & 4576 & & $52.0 \mathrm{~s}$
\end{tabular}

(232Cd, 235Se) $\mathrm{n}=75,645$

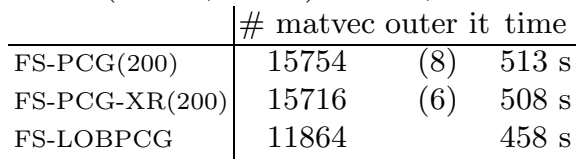

\begin{tabular}{l|ccc}
\multicolumn{4}{c}{$(83 \mathrm{Cd}, 81 \mathrm{Se}) \mathrm{n}=34,143$} \\
& \# matvec outer it & time \\
\hline FS-PCG $(200)$ & 15096 & $(11)$ & $264 \mathrm{~s}$ \\
FS-PCG-XR(200) & 12174 & $(5)$ & $209 \mathrm{~s}$ \\
FS-LOBPCG & 10688 & & $210 \mathrm{~s}$
\end{tabular}

(534Cd, 527Se) n $=141,625$

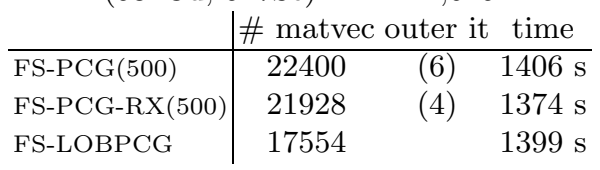

products than the PCG method, since the 5-point matrix-vector product takes approximately the time of 7 dot products, PCG gives a better timing.

The CG-XR method represents for this test case an interesting alternative for those two methods: it inherits the low number of matrix vector products from the LOBPCG and the low number of dot products from the PCG method.

\subsection{Numerical Results on Some Quantum Dots}

In this section we present numerical results on quantum dots up to thousand of atoms. The experiments are performed on the IBM-SP seaborg at NERSC.

For all the experiments we are looking for $\mathrm{mx}=10$ interior eigenvalues around $E_{r e f}=-4.8 \mathrm{eV}$, where the band gap is about 1.5 to $3 \mathrm{eV}$. We have calculated 4 typical quantum dots: (20Cd,19Se), (83Cd,81Se), (232Cd,235Se), (534Cd,527Se). These are real physical systems which can be experimentally synthesized and have been studied previously using the PCG method 10. Nonlocal pseudopotential is used for the potential term in equation (1), and spin-orbit interaction is also included. The cutoff energy for the plane-wave basis set is 6.8 Ryd. The stopping criterion for the eigenvector is $\|H x-x \lambda\| \leq$ tol $\|x\|$ where tol $=10^{-6}$. All the runs are performed on one node with 16 processors except for the smallest case $(20 \mathrm{Cd}, 19 \mathrm{Se})$ which is run on 8 processors. All the solvers are started with the same initial guess.

The preconditioner is the one given in [8]: diagonal with diagonal elements

$$
p_{i}=\frac{E_{k}^{2}}{\left.\left(\frac{1}{2} q_{i}^{2}+V_{0}-E_{r e f}\right)^{2}+E_{k}^{2}\right)},
$$

where $q_{i}$ is the diagonal term of the Laplacian, $V_{0}$ is the average potential and $E_{k}$ is the average kinetic energy of the wave function $\psi$. It is meant to be an approximation of the inverse of $\left(H-E_{r e f}\right)^{2}$. 
A notable fact is that all the solvers find the same 10 eigenvalues with the correct accuracy for all the runs. Therefore they are all robust.

The timing results are given in Table 4. For each test case the number of atoms of the quantum dot and the order $n$ of the corresponding matrix is given. The parameter for the number of iterations in the inner loop (nline) for FS-PCG and FS-PCG-XR is chosen to be the optimal one among the values 20, 50, 100, 200, and 500 and is given in brackets after the solver.

From Table 4, we observe that the three methods behave almost the same. The best method (in term of time) being either FS-PCG-XR or FS-LOBPCG.

FS-LOBPCG should also benefit in speed over FS-PCG and FS-PCG-XR from the fact that the matrix-vector products are performed by block. This is not the case in the version of the code used for this paper where the experiments are performed on a single node. The blocked implementation of FS-LOBPCG in PESCAN should run faster and also scale to larger processor counts as latency is less of an issue in the communications part of the code.

Another feature of FS-LOBPCG that is not stressed in Table 4 is its overwhelming superiority over FS-PCG when no preconditioner is available. In Table 5 Left, we illustrate this later feature. For the quantum dot $(83 \mathrm{Cd}, 81 \mathrm{Se})$, FS-LOBPCG runs 4 times faster than FS-PCG without preconditioner whereas it runs only 1.4 times faster with. For the four experiments presented in Table 4 the number of inner iteration that gives the minimum total time is always attained for a small number of outer iteration, this is illustrated in Table 5 Right for $(232 \mathrm{Cd}, 235 \mathrm{Se})$ where the minimum time is obtained for 6 outer iterations. Another and more practical way of stopping the inner iteration is in fixing the requested tolerance reached at the end of the inner loop. We call FS-PCG $(k)$ FS-PCG where the inner loop is stopped when the accuracy is less than $k^{n_{\text {outer }}}$, where $n_{\text {outer }}$ is number of the corresponding outer iteration. In Table 5 Right, we give the results for FS-PCG $\left(10^{-1}\right)$ and $(223 \mathrm{Cd}, 235 \mathrm{Se})$. It comes without a surprise that this solver converge in 6 outer steps. This scheme looks promising. It also allows a synchronized convergence of the block vectors.

Table 5. Left: Comparison of FS-PCG and FS-LOBPCG with and without preconditioner to find $\mathrm{mx}=10$ eigenvalues of the quantum dots $(83 \mathrm{Cd}, 81 \mathrm{Se})$; Right: The problem of finding the best inner length for FS-PCG can be avoided by fixing a tolerance as stopping criterion in the inner loop

(83Cd, 81Se) n $=34,143$

\begin{tabular}{ll|cc} 
& & \# matvec & time \\
\hline FS-PCG(200) & precond & 15096 & $264 \mathrm{~s}$ \\
FS-LOBPCG & precond & 10688 & $210 \mathrm{~s}$ \\
& & \\
FS-PCG(200) & no precond & 71768 & $1274 \mathrm{~s}$ \\
FS-LOBPCG & no precond & 17810 & $341 \mathrm{~s}$
\end{tabular}

(232Cd, 235Se) n $=75,645$

\begin{tabular}{l|ccc} 
& \# matvec outer it & time \\
\hline FS-PCG(100) & 17062 & $(15)$ & $577 \mathrm{~s}$ \\
FS-PCG(200) & 15716 & $(6)$ & $508 \mathrm{~s}$ \\
FS-PCG(300) & 15990 & $(4)$ & $517 \mathrm{~s}$ \\
& & & \\
FS-PCG $\left(10^{-1}\right)$ & 15076 & $(6)$ & $497 \mathrm{~s}$
\end{tabular}




\section{Conclusions}

In this paper, we described and compared 3 nonlinear CG methods with folded spectrum to find a small amount of interior eigenvalues around a given point. The application is to make a computational prediction of the electronic properties of quantum nanostructures. The methods were specifically selected and tuned for computing the electronic properties of large nanostructures. There is need for such methods in the community and the success of doing large numerical simulations in the field depend on them. All three methods are similar and thus often the results are close; a general ranking being: FS-LOBPCG is the fastest, next FS-PCG-XR and finally FS-PCG. In terms of memory requirement the three methods are ranked in the same way: FS-LOBPCG/FS-PCG-XR requires four/two times as much memory as FS-PCG. As our problem scales up the memory has not shown up as a bottleneck yet, i.e. using FS-LOBPCG is affordable.

The main drawback of FS-PCG and FS-PCG-XR is their sensitivity to the parameter nline (the number of iterations in the inner loop). In order to get rid of this parameter one can instead have a fixed residual reduction to be achieved on each step of the outer loop.

On other applications, the performance of FS-LOBPCG would be still better than FS-PCG if a fast block matrix-vector product and an accommodating preconditioner are available.

Finally, based on our results, if memory is not a problem and block version of the matrix-vector multiplication can be efficiently implemented, the FS-LOBPCG will be the method of choice for the type of problems discussed.

\section{Acknowledgements}

This research used resources of the National Energy Research Scientific Computing Center, which is supported by the Office of Science of the U.S. DOE.

\section{References}

1. Arbenz, P., Hetmaniuk, U.L., Lehoucq, R.B., Tuminaro, R.S.: A comparison of eigensolvers for large-scale 3D modal analysis using AMG-preconditioned iterative methods. Int. J. Numer. Meth. Engng (to appear)

2. Bai, Z., Demmel, J., Dongarra, J., Ruhe, A., van der Vorst, H. (Editors): Templates for the solution of Algebraic Eigenvalue Problems: A Practical Guide, SIAM, Philadelphia (2000)

3. Canning, A., Wang, L.W., Williamson, A., Zunger, A.: Parallel empirical pseudopotential electronic structure calculations for million atom systems. J. Comp. Phys. 160 (2000) 29-41

4. Knyazev, A.: Toward the optimal preconditioned eigensolver: locally optimal block preconditioned conjugate gradient method. SIAM J. on Scientific Computing 23(2) (2001) 517-541

5. Payne, M.C., Teter, M.P., Allan, D.C., Arias, T.A., Joannopoulos, J.D.: Iterative minimization techniques for ab initio total-energy calculations: molecular dynamics and conjugate gradients. Rev. Mod. Phys. 64 (1992) 1045-1097 
6. Thornquist, H.: Fixed-Polynomial Approximate Spectral Transformations for Preconditioning the Eigenvalue Problem. Masters thesis, Rice University, Department of Computational and Applied Mathematics (2003)

7. Wang, L.W., Li, J.: First principle thousand atom quantum dot calculations. Phys. Rev. B 69 (2004) 153302

8. Wang, L.W., Zunger, A.: Pseudopotential Theory of Nanometer Silicon Quantum Dots application to silicon quantum dots. In Kamat, P.V., Meisel, D.(Editors): Semiconductor Nanoclusters (1996) 161-207

9. Wang, L.W., Zunger, A.: Solving Schrodinger's equation around a desired energy: application to silicon quantum dots. J. Chem. Phys. 100(3) (1994) 2394-2397

10. Wang, L.W., Zunger, A.: Pseudopotential calculations of nanoscale CdSe quantum dots. Phys. Rev. B 53 (1996) 9579 